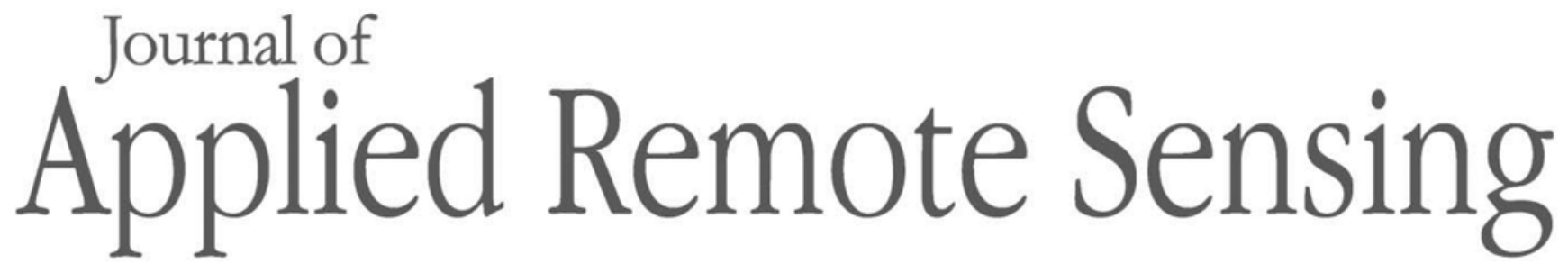

RemoteSensing.SPIEDigitalLibrary.org

\title{
Development of a simulation environment to support intercalibration studies over the Algodones Dunes system
}

Rehman S. Eon Aaron D. Gerace

Matthew Montanaro Brittany L. Ambeau Joel T. McCorkel 


\title{
Development of a simulation environment to support intercalibration studies over the Algodones Dunes system
}

\author{
Rehman S. Eon, ${ }^{\mathrm{a}, *}$ Aaron D. Gerace, ${ }^{\mathrm{a}}$ Matthew Montanaro, ${ }^{\mathrm{a}}$ \\ Brittany L. Ambeau, ${ }^{a}$ and Joel T. McCorkel ${ }^{b}$ \\ ${ }^{a}$ Rochester Institute of Technology, Rochester, New York, United States \\ bASA Goddard Space Flight Center, Greenbelt, Maryland, United States
}

\begin{abstract}
The ability of sensors to detect changes in the Earth's environment is dependent on retrieving radiometrically consistent and calibrated measurements from its surface. Intercalibration provides consistency among satellite instruments and ensures fidelity of scientific information. Intercalibration is especially important for spaceborne satellites without any on-board calibration, as accuracy of instruments is significantly affected by changes that occur postlaunch. To better understand the key parameters that impact the intercalibration process, this paper describes a simulation environment that was developed to support the primary mission of the Algodones Dunes campaign. Specifically, measurements obtained from the campaign were utilized to create a synthetic landscape to assess the feasibility of using the Algodones Dunes system as an intercalibration site for spaceborne instruments. The impact of two key parameters (differing view-angles and temporal offsets between instruments) on the intercalibration process was assessed. Results of these studies indicate that although the accuracy of intercalibration is sensitive to these parameters, proper knowledge of their impact leads to situations that minimize their effect. This paper concludes with a case study that addresses the feasibility of performing intercalibration on the International Space Station's platform to support NASA's CLARREO, the climate absolute radiance and refractivity observatory, mission. ( $)$ The Authors. Published by SPIE under a Creative Commons Attribution 3.0 Unported License. Distribution or reproduction of this work in whole or in part requires full attribution of the original publication, including its DOI. [DOI: 10.1117/1. JRS.12.012008]
\end{abstract}

Keywords: intercalibration; Digital Imaging and Remote Sensing Image Generation; simulation and modeling; BRDF; Ross-Li; Hapke; International Space Station.

Paper 170484SS received Jun. 2, 2017; accepted for publication Sep. 14, 2017; published online Oct. 6, 2017.

\section{Introduction}

The calibration of spaceborne sensors is critical to ensure continuity and accuracy in long-term studies of geophysical parameters. ${ }^{1}$ Over the past 40 years, there have been several Earth observing systems launched to measure changes in Earth's surface and atmosphere. ${ }^{2}$ Spaceborne sensors are continuously in development to ensure long-term studies of geophysical parameters, but inherent temporal gaps reduce the ability to monitor changes in the Earth's environment. Landsat- 8 represents the latest spaceborne satellite from the Landsat Data Continuity Mission. ${ }^{3}$ Landsat-8 has a spatial resolution of $30 \mathrm{~m}$, which was designed to support most environmental studies. However, Landsat- 8 provides global coverage every 16 days and, on average, has $35 \%$ of its images plagued by cloud cover. ${ }^{2,4}$ This inherent temporal gap limits the effectiveness for studying fine-scale changes in the Earth's surface and highlights the utility of intercalibration. ${ }^{2}$ Since intercalibration can combine measurements from two different sensors, this technique can be leveraged to improve spatial, spectral, and temporal coverage. ${ }^{2}$

*Address all correspondence to: Rehman S. Eon, E-mail: rse4949@rit.edu 
A joint experiment was conducted in March 2015 involving NASA Goddard Space Flight Center, the Rochester Institute of Technology (RIT), South Dakota State University, the University of Arizona, and the University of Lethbridge to support intercalibration studies over the Algodones Dunes system in Southern California. The Algodones site was chosen due to its potential to be used for vicarious calibration of different NASA satellite systems. The dunes system exhibits very similar characteristics to the well-known pseudoinvariant calibration site (PICS) Libya-4. ${ }^{5,6}$ Due to its accessibility, it is challenging to obtain ground-truth measurements for characterization of the landscape from PICS such as Libya-4, which is located in the Sahara Desert of North Africa. Alternatively, the Algodones Dunes system, located $2 \mathrm{~h}$ from San Diego in the United States, makes it significantly easier to perform the necessary field campaigns to characterize its terrain for the purpose of absolute calibration. ${ }^{5,6}$ The model-based studies performed here were designed to support a primary mission of the Algodones Dunes campaign to provide an insight into uncertainties that need to be accounted for when performing intercalibration.

The Digital Imaging and Remote Sensing Image Generation (DIRSIG) tool is a physicsbased synthetic image generation model developed by the RIT, ${ }^{7}$ which can be used to perform various intercalibration studies. The advantage of using a simulation and modeling software such as DIRSIG is that it provides the ability to vary parameters that affect the intercalibration process independently from one another. Although DIRSIG can model different spectral response functions, methods to correct for these differences are available in the works by Chander et al. ${ }^{8}$ The studies in this paper specifically investigate the lesser known effects of differing view geometries (sensor view and illumination angles) and time-of-collect on intercalibration. The simulations described in this paper were conducted to provide an understanding of how pseudoinvariant sites such as the Algodones Dunes can be used for intercalibration of satellite sensors and to help define the key factors that need to be considered.

The ability of DIRSIG to simulate spaceborne instruments was verified in this work by simulating the Aqua-moderate-resolution imaging spectroradiometer (MODIS) and Terra-MODIS sensors over the Algodones Dunes. The at-sensor radiance measurement of the simulations was compared to data collected by the actual sensors over the dunes system. The validation study utilizes band 1 ( 0.620 to $0.670 \mu \mathrm{m})$, band 4 ( 0.545 to $0.565 \mu \mathrm{m})$, and band $3(0.459$ to $0.479 \mu \mathrm{m})$ of the MODIS instrument.

The verification of the model was followed by an evaluation of DIRSIG to support intercalibration between sensors. The information provided by the MODIS sensor was used to simulate Landsat- 8 data. Band 2 ( 0.452 to $0.512 \mu \mathrm{m})$, band $3(0.5330 .590 \mu \mathrm{m})$, and band 4 (0.636 to $0.673 \mu \mathrm{m}$ ) of Landsat- 8 were simulated using DIRSIG and compared to cloud-free data collected by the actual sensor.

To support future NASA missions, this paper evaluates the potential to use International Space Station (ISS)-based imaging platforms for intercalibration. Specifically, an evaluation of the Solar, Lunar for Absolute Reflectance Imaging Spectroradiometer (SOLARIS), ${ }^{9,10}$ sensor for calibrating other spaceborne sensors such as MODIS and Landsat was conducted. SOLARIS is the reflected solar instrument of the climate absolute radiance and refractivity observatory (CLARREO) mission. ${ }^{9-11}$ The objective of the SOLARIS sensor is the development and inspection of calibration techniques, establish methods to obtain SI-traceability, and estimate reflectance from measurements of the sun and the scene. ${ }^{9}$ The mission plan is to have the SOLARIS sensor placed on the ISS platform to investigate techniques and benefits in obtaining highly accurate measurements before placing high-budget satellites into service. ${ }^{9}$ As a platform, the ISS has become an attractive option to perform intercalibration studies, as the long service life of the ISS along with the presence of a human crew and various equipment provide the possibility of performing various studies, which are not feasible with spaceborne satellites. ${ }^{12}$

The ISS intercalibration studies presented here utilize the Aqua-MODIS instrument as a test sensor. There are numerous parameters that potentially need to be considered to perform an accurate intercalibration between sensors [e.g., view geometry of the sensors, time-of-collect, differences in spectral response functions, and the bidirectional reflectance distribution function (BRDF) of the material]. These studies focused on assessing the impact of differing view geometries and time-of-collect on the intercalibration process. Specifically, the development of a simulated landscape and a forward modeling approach was utilized to assess the sensitivity of the intercalibration of Aqua-MODIS with SOLARIS on these two parameters. 


\section{Synthetic Landscape Development, Model Verification, and Intercalibration}

This section introduces the methodology used to create a synthetic landscape in DIRSIG. The process used to verify the DIRSIG software by simulating MODIS is detailed in Sec. 2.2, followed by an intercalibration study of Landsat-8 using Aqua-MODIS.

\subsection{Synthetic Landscape Development}

To leverage data collected from the ground-truth campaign of the Algodones Dunes during 2015, DIRSIG was used to study factors that need to be considered when performing intercalibration over pseudoinvariant sites such as Algodones. The region used to perform the intercalibration introduced in this work is a $5 \mathrm{~km} \times 5 \mathrm{~km}$ area with the center pixel located at $32^{\circ} 53^{\circ} 06^{\prime \prime} \mathrm{N}$ and $115^{\circ} 00^{\circ} 57^{\prime \prime} \mathrm{W}$. Leveraging the airborne and in situ measurements taken during the field campaign, DIRSIG was used to develop a synthetic, but realistic, landscape of Algodones. NASA Goddard's LiDAR, hyperspectral, and thermal (G-LiHT) sensor was extensively used to image the Algodones Dunes. ${ }^{13}$ The Goniometer of the RIT (GRIT) was also used to collect groundtruth data over the scene of interest. ${ }^{14}$ Details of these instruments can be found in the summary article for the field campaign. ${ }^{5}$ Figure 1(a) shows the region within Algodones used to create the simulated scene, whereas Fig. 1(b) shows the GRIT instrument taking measurements on the ground with the NASA G-LiHT flying overhead during the 2015 field campaign.

The synthetic scene of Algodones was created using the "scene construction tool" built into DIRSIG. ${ }^{15}$ This tool ingests various forms of image data to describe the terrain. Figure 2 shows the different sources of data/inputs required by the DIRSIG tool to describe the simulated landscape. High-resolution imagery by the National Agricultural Imagery Program (NAIP) dataset was used to classify the landscape and to describe its texture. ${ }^{16}$ The 2 -m digital elevation model provided by G-LiHT was used to facetize the geometric properties of the terrain. Hyperspectral data collected by GRIT during the field campaign were used to assign spectral features to each of the classes (defined by the NAIP data) within the synthetic landscape. The BRDF properties were described from the MODIS BRDF product, ${ }^{17}$ which uses the Ross-Li model ${ }^{18-21}$ It should be noted that this is not the most appropriate model to describe the BRDF properties for sand as this kernel-based model does not adequately capture the hot spot (or the backscatter direction). ${ }^{22}$ Future work will focus on incorporating actual BRDF measurements from the Algodones campaign into DIRSIG.

The DIRSIG simulation environment provides users with the ability to create sensor models at various focal lengths, sensor geometries, spectral responses, and platform motions, and to

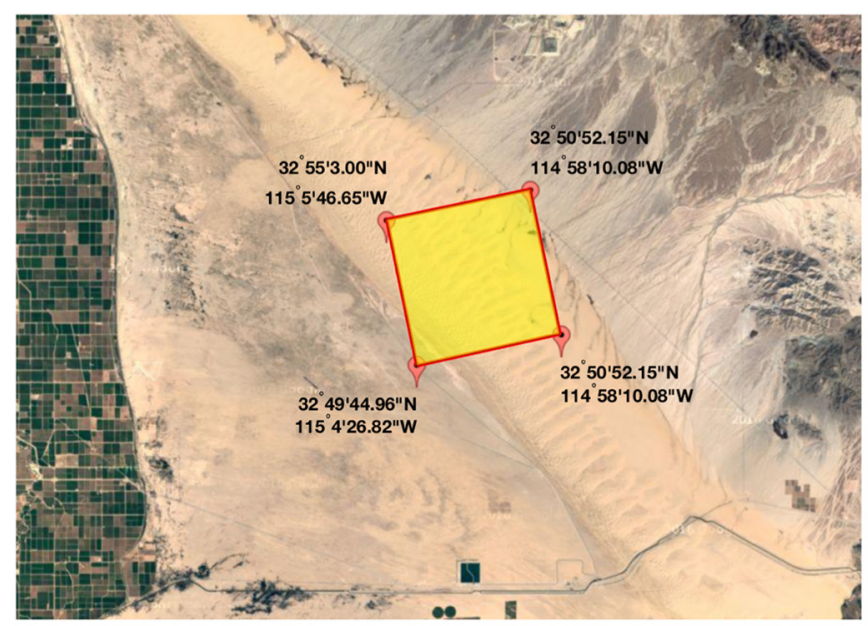

(a)

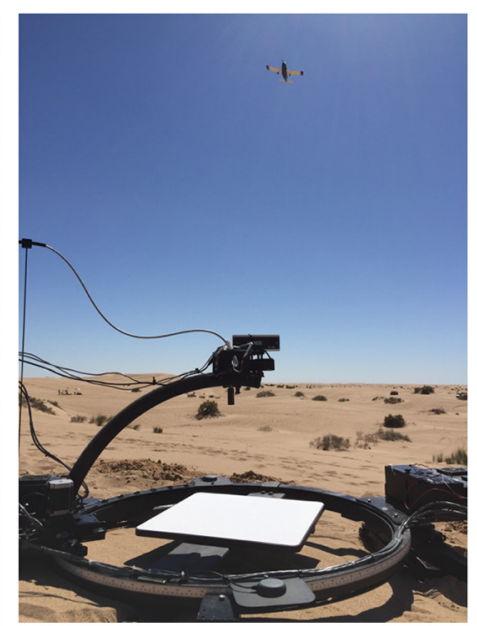

(b)

Fig. 1 (a) The $5 \mathrm{~km} \times 5 \mathrm{~km}$ region of interest in the Algodones Dunes used to create the synthetic landscape for DIRSIG. (b) The image shows the GRIT instrument and the NASA G-LiHT taking measurement of the dunes system during the 2015 field campaign. 


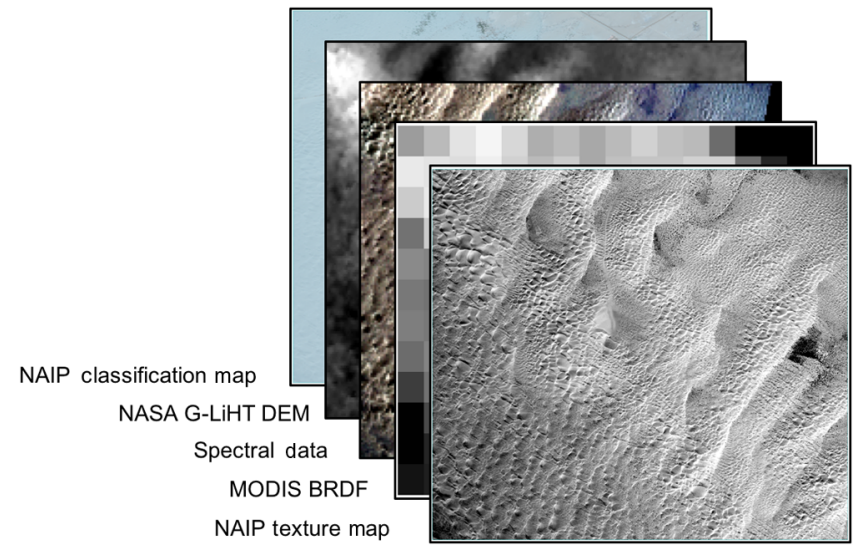

Fig. 2 The various data sources required by the DIRSIG construction tool to describe the synthetic landscape (Algodones Dunes).

(a)

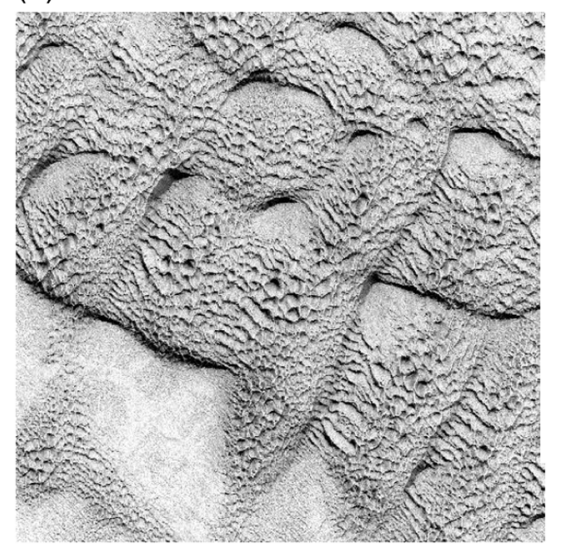

(b)

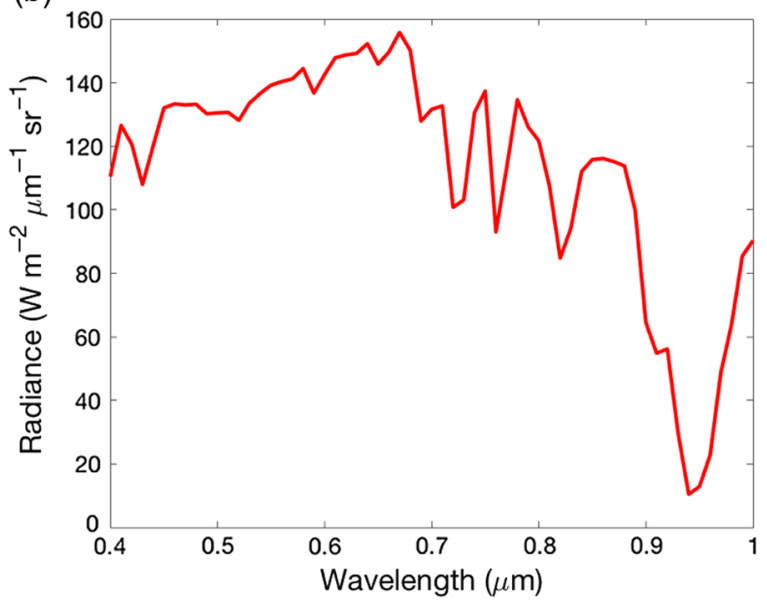

Fig. 3 (a) A nadir-looking image of the Algodones Dunes at a GSD of $10 \mathrm{~m}$ and (b) the subsequent average radiance of the scene in the VNIR.

view through various atmospheres. This allows the creation of any airborne or spaceborne sensors to facilitate intercalibration studies. Figure 3 shows a nadir-looking image of the Algodones scene that was developed with the "scene construction tool," and the subsequent top of atmosphere (TOA) radiance for an arbitrary pixel when imaging with a hyperspectral sensor model. The Algodones Dunes was imaged at a ground sample distance (GSD) of $10 \mathrm{~m}$ using a visible and near-infrared (VNIR) hyperspectral sensor (101 bands) over the 0.4-to 1.00- $\mu \mathrm{m}$ spectral range.

\subsection{Digital Imaging and Remote Sensing Image Generation Verification}

DIRSIG's potential to be used for intercalibration studies was performed by imaging the synthetic Algodones scene using different spaceborne satellite systems and comparing the TOA radiance to actual sensor data. The ability of DIRSIG to estimate/model TOA radiance of spaceborne sensors is dependent on an accurate representation of the scene and the atmosphere. Recall that the material properties of the scene were measured during the field-campaign so only a characterization of the atmospheric conditions is required to simulate the TOA radiance.

DIRSIG employs the radiative transfer algorithm moderate spectral resolution atmospheric transmittance model, ${ }^{23}$ or MODTRAN, to simulate atmospheric conditions. In this verification study, atmospheric conditions are estimated for a fixed Aqua-MODIS geometry $(\theta=188.58 \mathrm{deg}$ and $\phi=31.64 \mathrm{deg}$ ) using a standard midlatitude summer profile and a desert aerosol for 
several visibilities, which was compiled into a look-up-table (LUT). Then, to characterize (or estimate) the atmospheric conditions of Algodones for DIRSIG, actual MODIS data are compared to the LUT over an entire year for visibilities ranging from 20 to $50 \mathrm{~km}$ in 1-km increments. Note that the purpose of this study is to assess DIRSIG's ability to model at-sensor satellite radiance. So, a rigorous characterization of the atmosphere was not performed.

The LUT is shown in Fig. 4(a). The minimum percent difference (PD) between real and simulated data was used to estimate the atmospheric inputs that provided the best match. The minimum difference was observed at a visibility of $41 \mathrm{~km}$. Figure 4(b) shows the radiance measurement between the Aqua-MODIS and its simulation for the best fit. The root-mean-square error (RMSE) and PD for band 3, band 4, and band 1 were $12.23 \mathrm{Wm}^{-2} \mu \mathrm{m}^{-1} \mathrm{sr}^{-1}(\mathrm{PD}=$ $1.26 \%), 9.19 \mathrm{Wm}^{-2} \mu \mathrm{m}^{-1} \mathrm{sr}^{-1}(\mathrm{PD}=5.14 \%)$, and $14.70 \mathrm{Wm}^{-2} \mu \mathrm{m}^{-1} \mathrm{sr}^{-1}(\mathrm{PD}=8.81 \%)$, respectively. The observed residuals are reasonable considering the use of Ross-Li and the nature in which the atmosphere was estimated.

With an estimate of the atmosphere in place, the TOA radiance for two spaceborne sensors was simulated: Aqua-MODIS and Terra-MODIS. Specifically, bands 1, 4, and 3 of the MODIS sensors were simulated and compared to real cloud-free data. The MODIS sensors were simulated for four different view geometries from 2012 to 2015, see Table 1.

Figure 5 compares the average radiance of the scene between the actual and simulated TerraMODIS for band 3, band 4, and band 1 (the RGB bands) of MODIS. The results for four different view geometries of Aqua-MODIS are shown in Fig. 6. The RMSE and PD between the simulated and original data are summarized in Table 2. DIRSIG primarily captures the at-sensor variability for all the simulated view geometries of Terra and Aqua. An exception to this can be seen in Fig. 6, where the predicted radiance is significantly higher for a particular sensor geometry $(\theta=184.73 \mathrm{deg}, \phi=59.35 \mathrm{deg})$ of Aqua-MODIS. Considering that this is the longest slant path, this is likely due to the nature in which the atmosphere was estimated. The PD error was also significantly higher (greater than 50\%) for a few particular days. By inspection, it was determined that the TOA radiance from these datasets was affected by the presence of
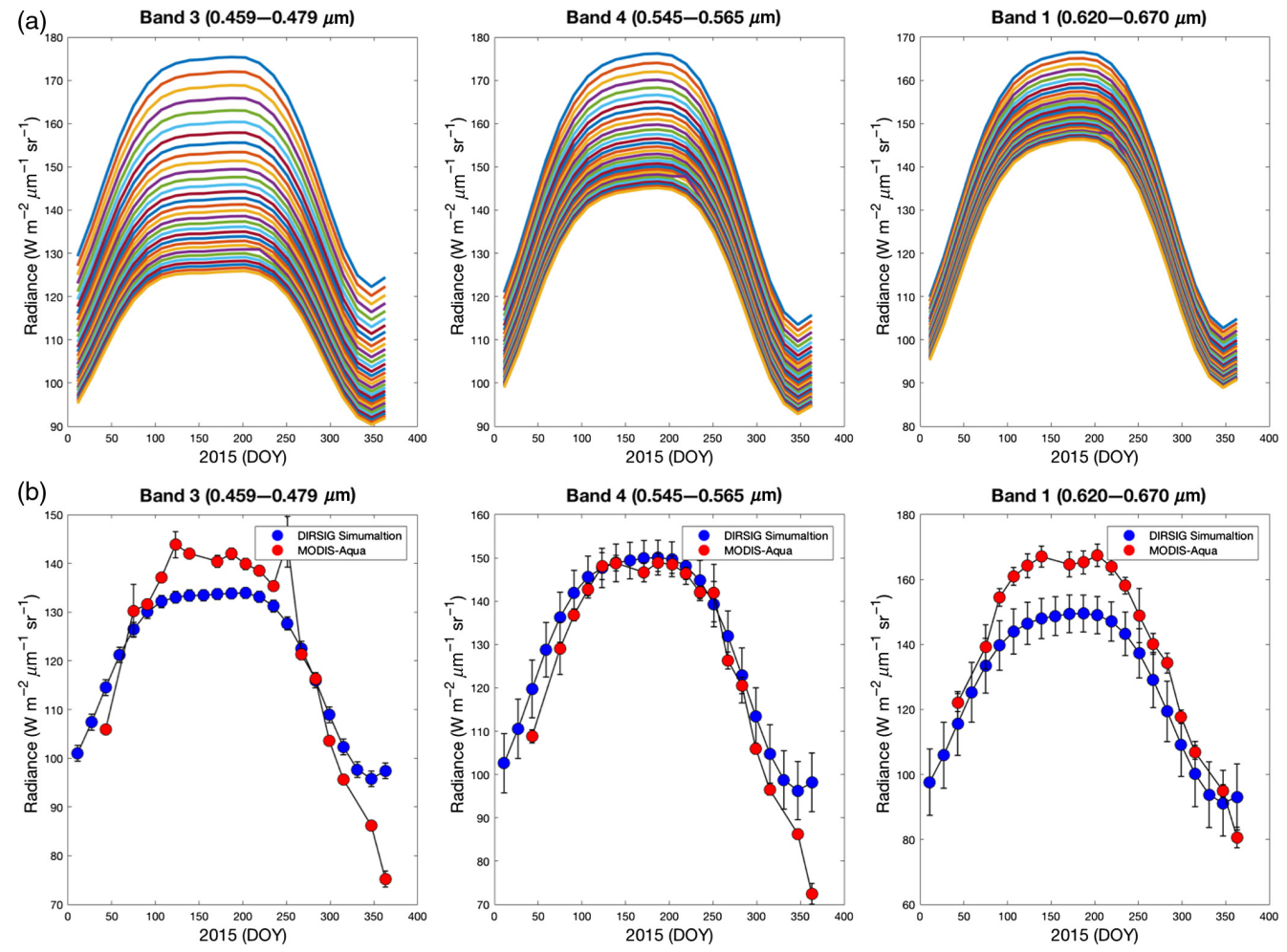

Fig. 4 (a) Aqua-MODIS with sensor azimuth and zenith of 188.58 deg and 31.64 deg, respectively, imaging the Algodones Dunes simulated over 2015 at visibilities ranging from 20 to $50 \mathrm{~km}$. (b) The best possible match of the simulated data with real measurements by the Aqua-MODIS. These measurements were observed at a visibility of $41 \mathrm{~km}$. 
Table 1 The different sensor view geometries of Terra-MODIS, Aqua-MODIS, and Landsat-8 being simulated in DIRSIG. The MODIS sensors are simulated from 2012 to 2015, whereas Landsat-8 is simulated from 2013 to 2017 . These simulated measurements are then compared to measurements taken by the real sensors over the Algodones Dunes.

\begin{tabular}{lcc}
\hline \hline & \multicolumn{2}{c}{ Sensor view geometry } \\
\cline { 2 - 3 } Sensor & Sensor azimuth $(\theta)(\mathrm{deg})$ & Sensor zenith $(\phi)(\mathrm{deg})$ \\
\hline Terra-MODIS & 352.7 & 39.8 \\
& 351.5 & 30.3 \\
& 347.9 & 7.3 \\
Aqua-MODIS & 167.8 & 5.3 \\
& 13.9 & 3.5 \\
& 188.9 & 9.0 \\
& 188.9 & 31.6 \\
Landsat-8 & 184.7 & 59.4 \\
& 352.0 & 15.0 \\
\hline \hline
\end{tabular}
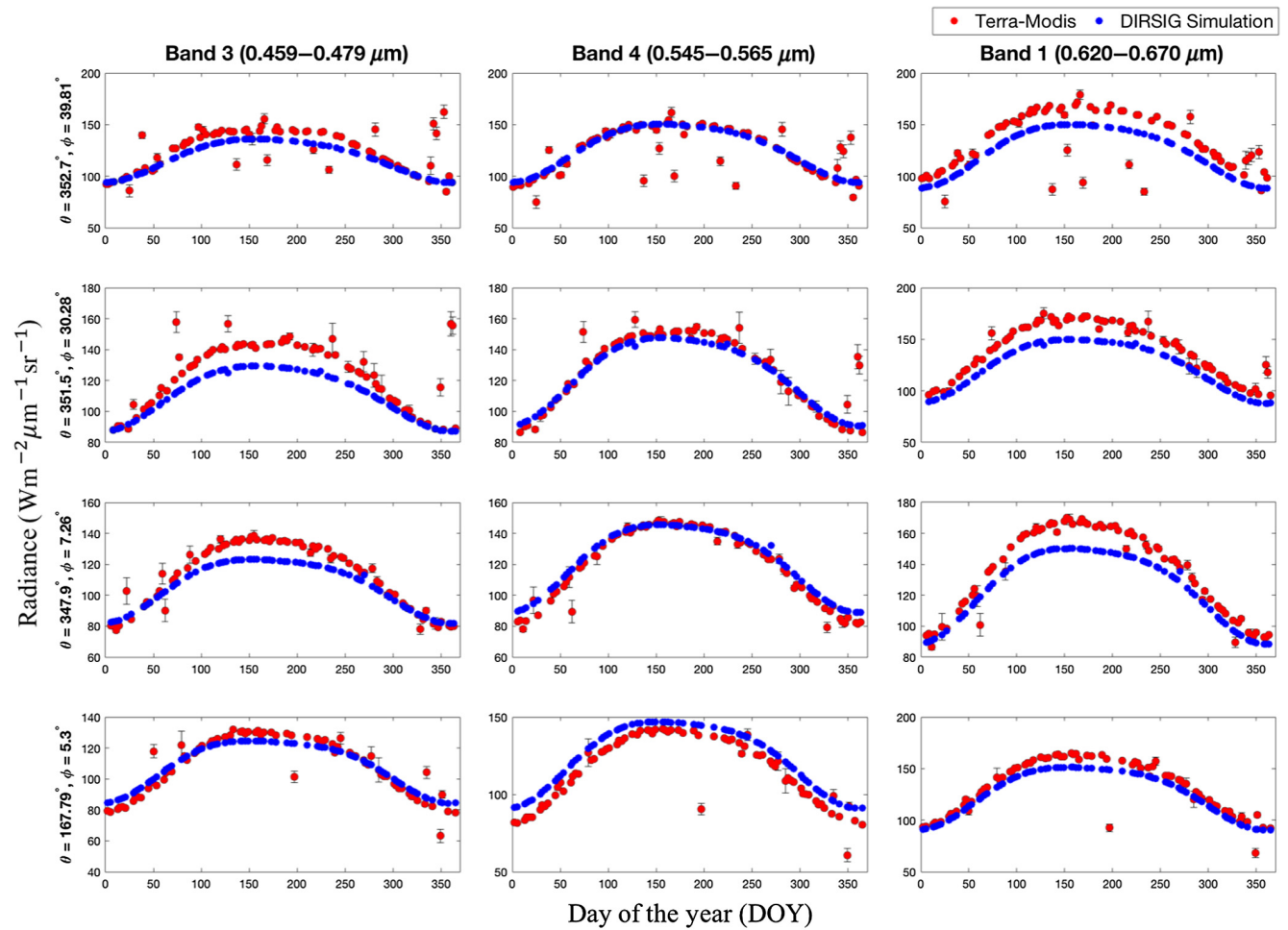

Fig. 5 The mean radiance calculated over the scene of interest in the Algodones Dunes using the Terra-MODIS sensor from 2012 to 2015 , which were compared to measurements simulated by DIRSIG. The radiance measurements were compared for the RGB bands of MODIS for four different sensor positions. The view geometries being simulated for the MODIS sensor is illustrated in Table 1. 
Table 2 The RMSE and PD with the DIRSIG simulations.

\begin{tabular}{|c|c|c|c|}
\hline \multirow[b]{2}{*}{ Sensor view geometry } & \multicolumn{3}{|c|}{ RMSE $\left(\mathrm{Wm}^{-2} \mu \mathrm{m}^{-1} \mathrm{sr}^{-1}\right)$ [PD (\%)] } \\
\hline & $\begin{array}{l}\text { Band } 3(0.459 \\
\text { to } 0.479 \mu \mathrm{m})\end{array}$ & $\begin{array}{l}\text { Band } 4(0.545 \\
\text { to } 0.565 \mu \mathrm{m})\end{array}$ & $\begin{array}{l}\text { Band } 1(0.620 \\
\text { to } 0.670 \mu \mathrm{m})\end{array}$ \\
\hline \multicolumn{4}{|c|}{ Terra-MODIS } \\
\hline$\theta=352.7 \mathrm{deg}, \phi=39.81 \mathrm{deg}$ & $7.66[4.94]$ & $2.33[0.01]$ & $15.80[11.40]$ \\
\hline$\theta=351.5 \mathrm{deg}, \phi=30.28 \mathrm{deg}$ & $10.98[7.71]$ & $3.32[0.87]$ & $15.49[10.70]$ \\
\hline$\theta=347.9 \mathrm{deg}, \phi=7.26 \mathrm{deg}$ & $8.57[5.38]$ & $4.76[2.63]$ & $11.82[7.91]$ \\
\hline$\theta=167.79 \mathrm{deg}, \phi=5.3 \mathrm{deg}$ & $4.89[0.50]$ & $8.27[7.02]$ & $7.84[5.30]$ \\
\hline \multicolumn{4}{|c|}{ Aqua-MODIS } \\
\hline$\theta=13.91 \mathrm{deg}, \phi=3.53 \mathrm{deg}$ & $5.85[0.84]$ & $8.42[7.09]$ & $8.35[5.46]$ \\
\hline$\theta=188.85 \mathrm{deg}, \phi=9.02 \mathrm{deg}$ & $7.50[1.62]$ & $6.81[4.60]$ & $11.11[6.85]$ \\
\hline$\theta=188.58 \mathrm{deg}, \phi=31.64 \mathrm{deg}$ & $6.52[0.25]$ & 6.14 [3.99] & $12.01[7.94]$ \\
\hline \multirow[t]{2}{*}{$\theta=184.73 \mathrm{deg}, \phi=59.35 \mathrm{deg}$} & $21.91[16.58]$ & 27.86 [22.32] & $10.61[7.23]$ \\
\hline & $\begin{array}{c}\text { Band } 2(0.450 \text { to } \\
0.510 \mu \mathrm{m})\end{array}$ & $\begin{array}{c}\text { Band } 3(0.530 \text { to } \\
0.590 \mu \mathrm{m})\end{array}$ & $\begin{array}{c}\text { Band } 4(0.640 \text { to } \\
0.670 \mu \mathrm{m})\end{array}$ \\
\hline \multicolumn{4}{|c|}{ Landsat-8 } \\
\hline$\theta=352.0 \mathrm{deg}, \phi=15.0 \mathrm{deg}$ & $7.21[5.31]$ & $12.98[11.10]$ & $7.11[4.58]$ \\
\hline$\theta=39.0 \mathrm{deg}, \phi=15.0 \mathrm{deg}$ & $6.94[1.60]$ & $10.30[8.11]$ & $6.02[2.31]$ \\
\hline
\end{tabular}

clouds within the scene, which was not modeled in DIRSIG. Most of the deviation between the simulated and original data occurred during the middle of the year, where the sun is typically at higher elevation. This is likely due to the limitation of the Ross-Li model to account for the hot spot effect. The hot spot or the opposition effect results in a sharp increase in reflectance in the backscatter direction. ${ }^{22,24}$ The original MODIS data have higher radiance during the middle of the year due to the hot spot effect, which the model does not capture with the Ross-Li input. Overall, the PDs are within a reasonable range for data to support the sensitivity studies presented in Sec. 3. However, future work will focus on driving down these errors to support intercalibration studies (presented next) by better characterizing the atmosphere and the BRDF of the Algodones Dunes.

\subsection{Intercalibration Study with Landsat-8}

The two MODIS sensors in Sec. 2.2 were used to verify the DIRSIG model's capability to adequately simulate imagery of the Algodones Dunes system. The feasibility of DIRSIG to perform intercalibration studies between two sensors is evaluated here by simulating Landsat- 8 using the aforementioned estimates of the material properties and the atmosphere from the MODIS sensor. Bands 2, 3, and 4 of Landsat- 8 were simulated and compared to real cloudfree data over the Algodones Dunes. Landsat- 8 was simulated for two view geometries from 2013 to 2017 (the sensor angles are reported in Table 1).

Figure 7 compares the average at-sensor radiance between the Landsat- 8 sensor and the simulation for two view geometries for band 2, band 3, and band 4. The RMSEs and PDs are summarized in Table 2. There is, again, good agreement between the original and simulated data. Some of the high PDs are due to the presence of clouds in the Landsat imagery, while other errors can be attributed to the estimates of the atmospheric and BRDF inputs in the DIRSIG model.

This study was designed to assess the feasibility of using DIRSIG as a transfer mechanism to intercalibrate one sensor with another. While the absolute errors are quite high to justify 

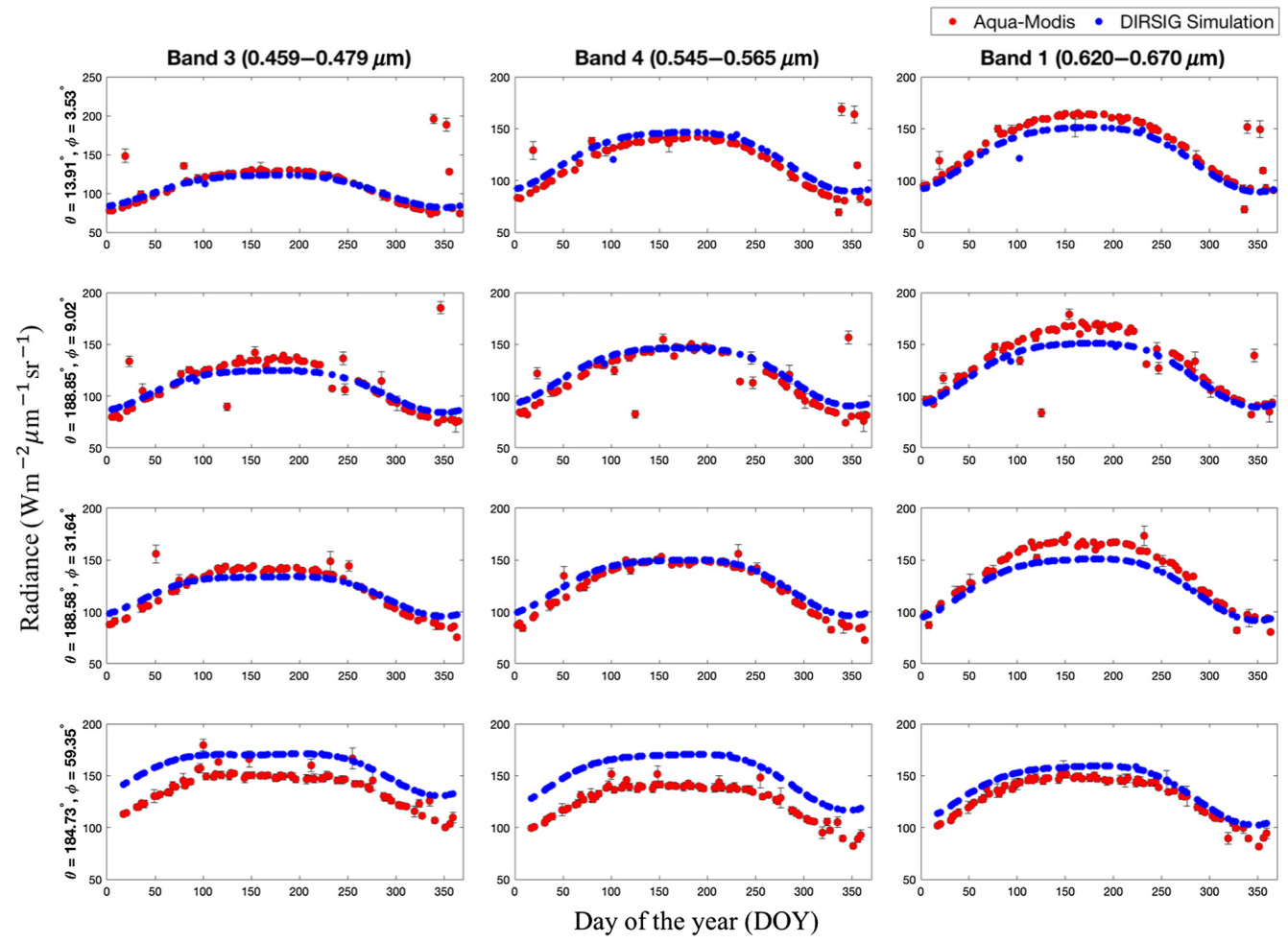

Fig. 6 The mean radiance calculated over the scene of interest in the Algodones Dunes using the Aqua-MODIS sensor from 2012 to 2015 , which were compared to measurements simulated by DIRSIG. The radiance measurements were compared for the RGB bands of MODIS for four different sensor positions. The view geometries being simulated for the MODIS sensor is illustrated in Table 1.
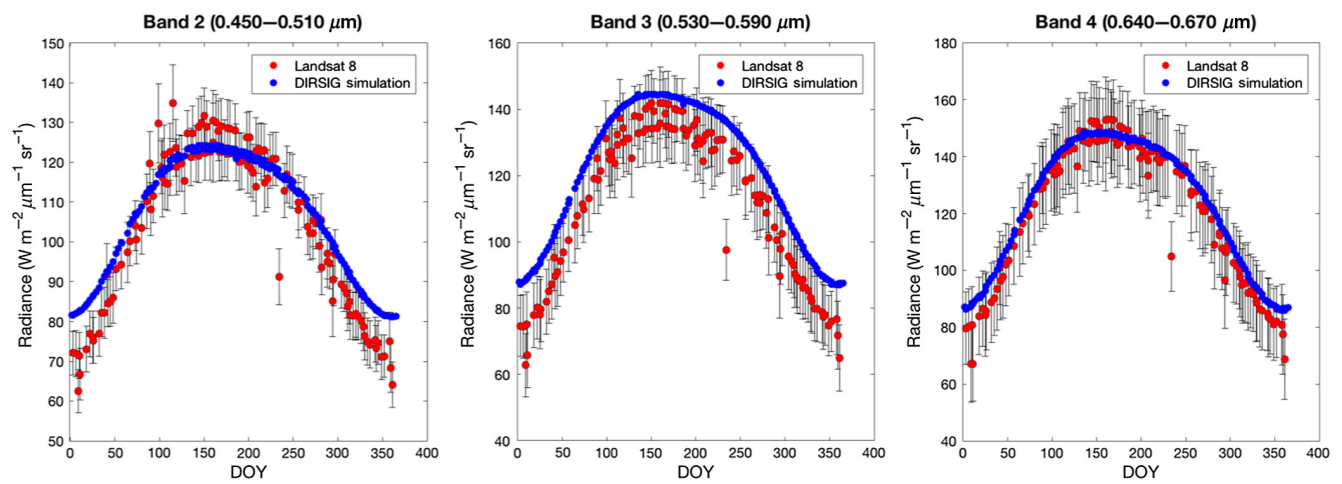

Fig. 7 The mean radiance calculated over the scene of interest in the Algodones Dunes using the Landsat-8 sensor from 2013 to 2017, which were compared to measurements simulated by DIRSIG. The radiance measurements were compared for the RGB bands of Landsat for its two different looks of the dunes system.

performing intercalibration of Landsat- 8 with MODIS-Aqua as described here, future work will assess the sensitivity of the atmospheric and BRDF inputs in intercalibration accuracy.

\section{Intercalibration Studies Using the International Space Station}

The focus of the March 2015 campaign was to evaluate the potential of pseudoinvariant sites such as the Algodones Dunes to support intercalibration studies of the CLARREO Pathfinder mission, specifically for the SOLARIS sensor. This section discusses methodologies that focus 
(a)

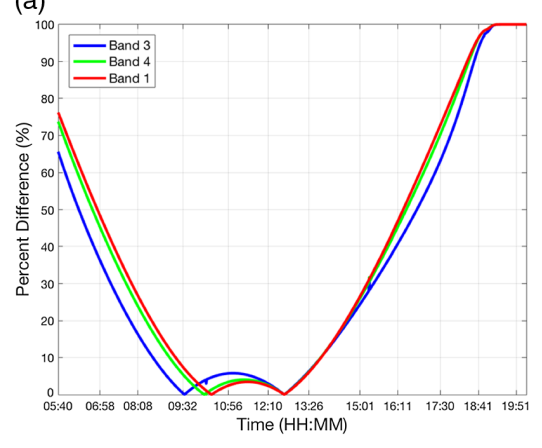

(b)

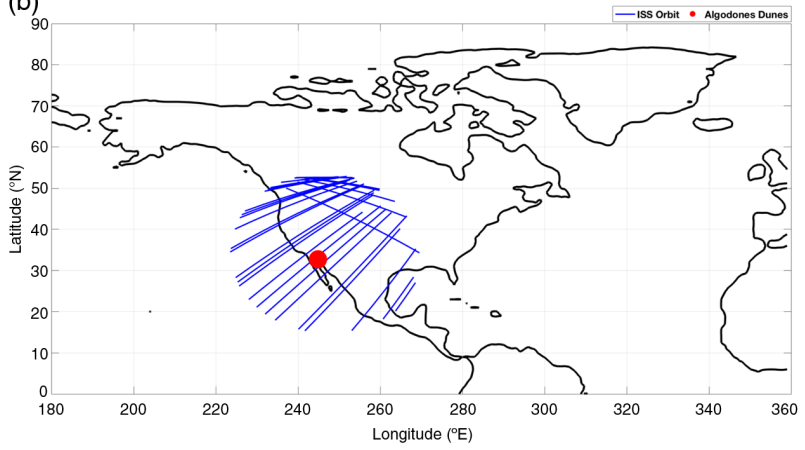

Fig. 8 (a) PD in the observed at-sensor radiance to the original MODIS-flight from sunrise to sunset. The PD is shown for bands 1,3 , and 4 of MODIS. The PD is relatively low $\pm 1 \mathrm{~h}$ of the initial flight time. (b) Intercalibration opportunities of Aqua-MODIS using a sensor, on board the ISS, over a 36-day period in June of 2014 based on the time constraint.

on assessing the potential to use instruments on-board the ISS to calibrate other spaceborne satellites. The Aqua-MODIS sensor was used as a test case in this work. The intercalibration study assesses the sensitivity of collection time (Sec. 3.1) and view geometry (Sec. 3.2) on intercalibration. Section 3.3 explores if the changing orbit of the ISS can cause any issues with intercalibration.

\subsection{Temporal Study}

Due to the nature of the intercalibration process, there will always be a temporal offset between sensors. To assess the impact of temporal offset on intercalibration accuracy, an Aqua-MODIS image was simulated for June 14, 2014 at 20:40 UTC over the Algodones Dunes using DIRSIG (note that this date/time represents an actual MODIS overpass). Next, the synthetic Algodones Dunes landscape was imaged from 13:40 to 04:10 UTC (sunrise to sunset) using DIRSIG. The difference in radiance between each simulated time and the nominal time was calculated to assess the impact of temporal offset of image acquisition on at-sensor radiance, which can be used to set the requirements for a temporal constraint for intercalibration opportunities.

The PD in the at-sensor radiance measurement between the nominal and test collection time for band 1, band 4, and band 3 of MODIS is shown in Fig. 8(a). The general trend in the plot

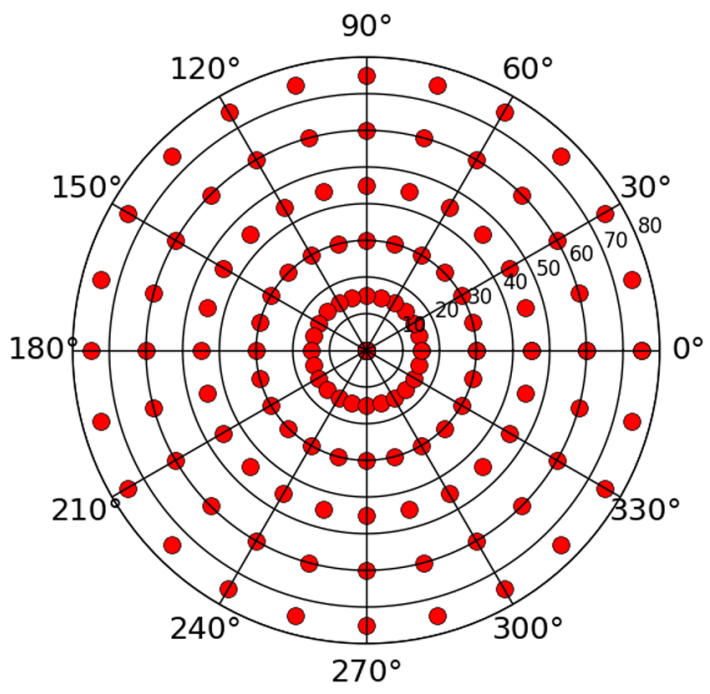

Fig. 9 DIRSIG is used to image the Algodones Dunes at various different view geometries for the Aqua-MODIS sensor. This was used to study the effect view geometry can have on the intercalibration process. 


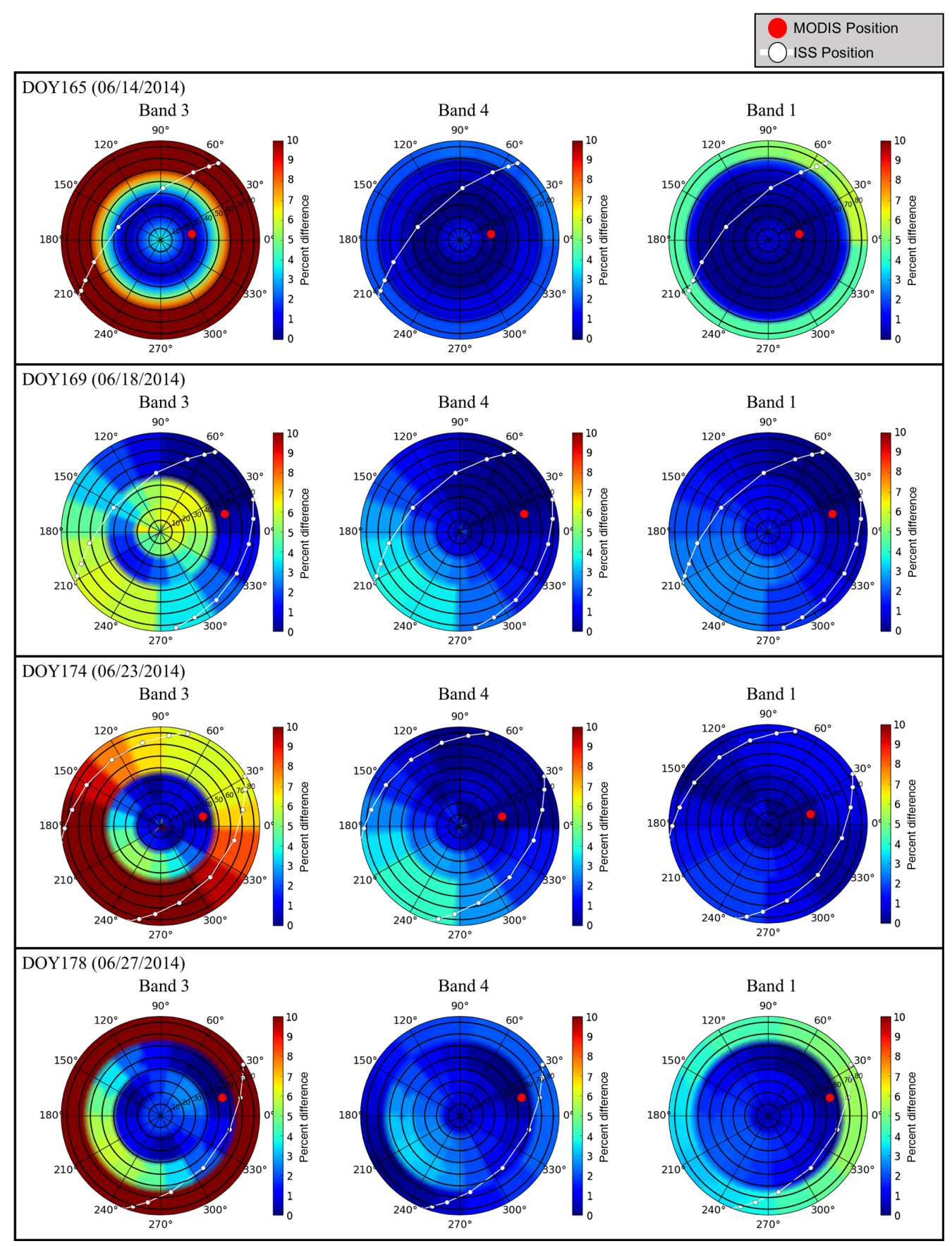

Fig. 10 The polar-plot illustrates the PD in the view geometry of the MODIS sensor in bands 3, 4, and 1 for four different days in June 2014 (DOY165, DOY169, DOY174, and DOY178).

indicates that the PD naturally increases with elapsed time from the nominal collect. However, there seems to be a small window of time, $\sim \pm 1 \mathrm{~h}$ of the original flight, where the PD is $\sim 5 \%$ or less for all three bands. Note that this time window is dependent on the amount of intercalibration accuracy required by the user's application. This \pm 1 -h time frame can potentially be used as a temporal constraint for intercalibration opportunities of the Aqua-MODIS with sensors on-board the ISS, while imaging the dunes system. The Systems Tool Kit (STK) from Analytical Graphics, Inc. was used to track the orbit of the ISS and find intercalibration opportunities over the 36-day period (May 29, 2014 to July 3, 2014) based on the temporal constraint. The Aqua-MODIS sensor typically images the Algodones Dunes between 20:00 UTC-21:35 UTC every 1 to 2 days, and based on the \pm 1 -h time constraint there were several days 


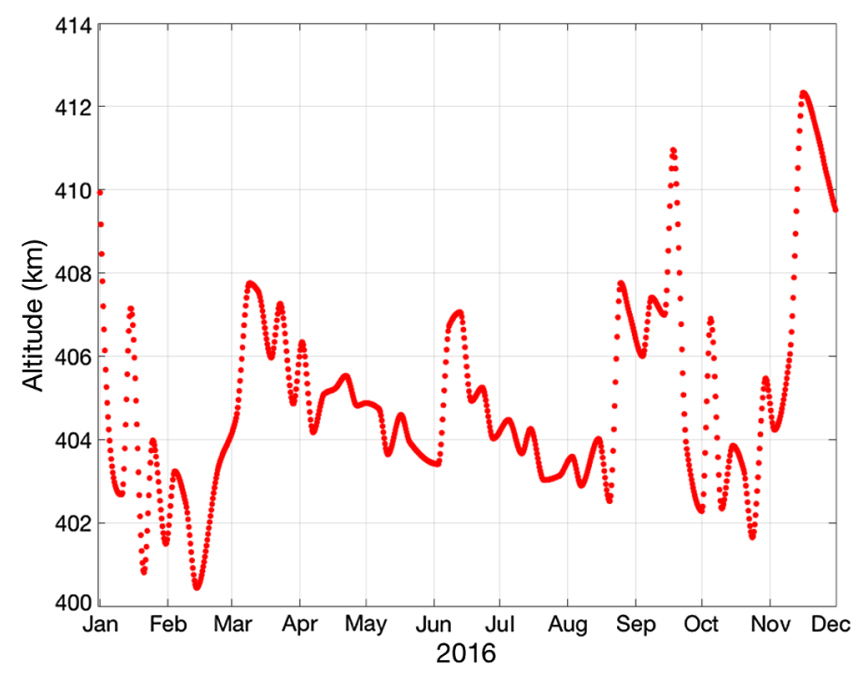

Fig. 11 The mean height $(\mathrm{km})$ of the ISS in 2016 over the Algodones Dunes. The changes in height are due to the orbital decay of the space station and subsequent reboost to maintain the orbit.

where intercalibration studies could be performed over the time period. Figure 8(b) shows the ISS groundtrack with the intercalibration opportunities over the 36-day period.

\subsection{View Geometry Study}

The view the geometry of a sensor has a significant effect on the at-sensor radiance measurements. The ISS and the Aqua platforms are in dramatically different orbits, and the likelihood of SOLARIS and MODIS having the same view geometries is low. Note that SOLARIS does have a three-axis gimble to minimize view angle differences, but they will still exist operationally. ${ }^{10}$ To quantify the impact of view geometry on the intercalibration accuracy, DIRSIG was used to simulate the Algodones Dunes at various geometries over a hemisphere $(\theta: 0$ deg to $360 \mathrm{deg}$ and $\phi: 0 \mathrm{deg}$ to $80 \mathrm{deg}$ ). The different view geometries used to image the Algodones Dunes for Aqua-MODIS sensor are shown in Fig. 9.

The PD in radiance of each view geometry from the nominal MODIS view was calculated for band 1, band 3, and band 4 for DOY165 (June 14, 2014), DOY169 (June 18, 2014), DOY174 (June 23, 2014), and DOY178 (June 27, 2014), where DOY is day of the year. The PDs are represented as polar plots in Fig. 10. The nominal MODIS view is shown in red, while the orbit of the ISS looking at the scene of interest within the Algodones Dunes is represented by the white curves within the polar plots.

The maximum difference due to the sensor position is $\sim 10 \%$, with no error when they have the same view geometry. Like the temporal study, sensor geometry constraints can be placed based on the user's application. There are a few different intercalibration opportunities for a sensor on-board the ISS (white curve) on each of the days shown in Fig. 10, where difference due to view geometry is low (e.g., less than 1\%) enough that it would not have had a significant effect on the calibration procedure. For example, in DOY 169, there seems to be $\sim 12$ calibration opportunities between the sensor on-board the ISS and the Aqua-MODIS sensor. The PD is less than $1 \%$ at those 12 different view geometries (white curve), indicating that this was a potentially good opportunity to perform intercalibration between the SOLARIS and Aqua-MODIS sensor. DOY174 represents a poor case for intercalibration, as the sensor on-board the ISS would have been in positions where the PD was well over $8 \%$ in band 3 of MODIS.

\subsection{Changing International Space Station Orbit}

The intercalibration opportunities in the two different studies were predicted based on dates in 2014, where both the orbits of ISS and Aqua-MODIS were known. Although, the orbit of 
Aqua-MODIS remains constant at an altitude of $705 \mathrm{~km}$, the same cannot be said about the ISS. ${ }^{25,26}$ The ISS resides in a lower earth orbit and experiences constant aerodynamic drag from the atmosphere. ${ }^{26,27}$ The orbit of the ISS is constantly decaying due to microgravity and requires periodic reboost to maintain the correct altitude. ${ }^{26,27}$ The reboosting maneuver is typically performed using thruster firings by the attached Progress $M$ spacecraft, which makes frequent trips to resupply the space station. ${ }^{27}$ The altitude profile of the ISS in 2016 over the Algodones Dunes, measured using the STK software, is shown in Fig. 11. The change in altitude of the ISS can be seen over the 1-year period; the negative slope is due to the orbital decay caused by the aerodynamic drag, whereas the sharp positive slopes are a result of the reboosting maneuvers. However, the ISS height changes only $12 \mathrm{~km}$ over the 1-year period, and the effect it has on the view geometry of a sensor, such as MODIS, is almost negligible. For all the view geometries of both Aqua- and Terra-MODIS, the biggest change in sensor zenith was $1 \mathrm{deg}$ due to change in height of the ISS. A change in sensor zenith of 1-deg affects the TOA radiance by $\sim 2 \mathrm{Wm}^{-2} \mu \mathrm{m}^{-1} \mathrm{sr}^{-1}$, which is quite negligible. So, the unpredictable nature of the ISS orbit should not influence intercalibration.

\section{Summary and Conclusion}

The objective of this paper was to develop a simulated environment to support intercalibration studies for the Algodones Dunes system. The first part of the work focused on the feasibility of the DIRSIG software to simulate spaceborne sensors. DIRSIG was used to simulate TOA radiance of the Algodones Dunes using Aqua-MODIS, Terra-MODIS, and Landsat-8. The TOA radiance was reported for the RGB bands of the sensors. Comparisons between simulated and original data for the three different sensors show good agreement, illustrating the potential utility of DIRSIG to serve as an intercalibration transfer mechanism between two instruments. Future work will focus on improving simulations by replacing the Ross-Li BRDF model with the radiative transfer equations developed by Hapke. ${ }^{28}$ Hapke's model has been widely used to estimate the BRDF of materials, especially for granular sediments. The BRDF model can take into account single scattering, multiple scattering events, as well as the opposition effects, which are the shadow hiding opposition effect and the coherent backscatter opposition effect. This would immensely improve DIRSIG's ability to simulate airborne and spaceborne satellites.

This work assessed some of the limiting factors in the intercalibration process. This paper investigated how differences in view geometries (sensor view and illumination angles) and timeof-collect can affect intercalibration with an ISS-based platform such as SOLARIS. In this specific study, it was demonstrated that a \pm 1 -h window from the nominal collect resulted in less than $5 \%$ differences for all three bands. This \pm 1 -h time frame can potentially be used as an initial temporal constraint for finding calibration opportunities between Aqua-MODIS and sensors onboard the ISS. Once a temporal constraint is determined, a view geometry constraint can be placed to minimize view angle effects. In the simulations presented here, maximum difference due to the sensor position was $\sim 10 \%$. Within the four different days used in this study, there were multiple calibration opportunities between a sensor on the ISS and Aqua-MODIS, where view geometry had less than $1 \%$ impact on the TOA radiance. Considering the versatility of the proposed SOLARIS gimble, these days represent adequate opportunities to perform intercalibration. This paper also explored the challenges that may arise from the ISS being present in a lower earth orbit. However, the change in the ISS orbit is not significant enough to have much of an influence on the intercalibration process.

\section{References}

1. C. N. Rao, C. Cao, and N. Zhang, "Inter-calibration of the moderate-resolution imaging spectroradiometer and the alongtrack scanning radiometer-2," Int. J. Remote Sens. 24(9), 1913-1924 (2003).

2. B. L. Ambeau et al., "The characterization of a DIRSIG simulation environment to support the inter-calibration of spaceborne sensors," Proc. SPIE 9972, 99720M (2016). 
3. J. R. Irons, J. L. Dwyer, and J. A. Barsi, "The next Landsat satellite: the Landsat Data Continuity Mission,” Remote Sens. Environ. 122, 11-21 (2012).

4. D. P. Roy et al., "Multi-temporal MODIS-Landsat data fusion for relative radiometric normalization, gap filling, and prediction of Landsat data," Remote Sens. Environ. 112(6), 3112-3130 (2008).

5. J. McCorkel et al., "Overview of the 2015 Algodones sand dunes field campaign to support sensor intercalibration,” J. Appl. Remote Sens. 12(1), 012003 (2017).

6. D. Helder et al., "Absolute radiometric calibration of Landsat using a pseudo invariant calibration site," IEEE Trans. Geosci. Remote Sens. 51(3), 1360-1369 (2013).

7. A. A. Goodenough and S. D. Brown, "DIRSIG 5: core design and implementation," Proc. SPIE 8390, 83900H (2012).

8. G. Chander et al., "Applications of spectral band adjustment factors (SBAF) for crosscalibration," IEEE Trans. Geosci. Remote Sens. 51(3), 1267-1281 (2013).

9. K. Thome et al., "Test plan for a calibration demonstration system for the reflected solar instrument for the climate absolute radiance and refractivity observatory," Proc. SPIE $\mathbf{8 5 1 6}$, 851602 (2012).

10. K. Thome, J. McCorkel, and B. McAndrew, "Demonstrating the error budget for the climate absolute radiance and refractivity observatory through solar irradiance measurements," Proc. SPIE 9607, 96071C (2015).

11. B. A. Wielicki et al., "Achieving climate change absolute accuracy in orbit," Bull. Am. Meteorol. Soc. 94(10), 1519-1539 (2013).

12. C. Roithmayr et al., "Opportunities to intercalibrate radiometric sensors from International Space Station," J. Atmos. Oceanic Technol. 31(4), 890-902 (2014).

13. B. D. Cook et al., "NASA Goddard's LiDAR, hyperspectral and thermal (G-LiHT) airborne imager," Remote Sens. 5(8), 4045-4066 (2013).

14. C. M. Bachmann et al., "Modeling geophysical properties of the Algodones Dunes from field and laboratory hyperspectral goniometer measurements using GRIT and comparison with G-LiHT imagery," Proc. SPIE 9972, 99720K (2016).

15. A. D. Gerace et al., "The development of a DIRSIG simulation environment to support instrument trade studies for the SOLARIS sensor," Proc. SPIE 9472, 947214 (2015).

16. United States Department of Agriculture: Farm Service Agency, https:/www.fsa.usda.gov/ programs-and-services/aerial-photography/imagery-programs/naip-imagery/ (2017).

17. C. B. Schaaf et al., "First operational BRDF, albedo nadir reflectance products from MODIS," Remote Sens. Environ. 83(1), 135-148 (2002).

18. J. K. Ross, The Radiation Regime and Architecture of Plant Stands, Dr. w. Junk, The Hague, The Netherlands (1981).

19. X. Li and A. H. Strahler, "Geometric-optical bidirectional reflectance modeling of the discrete crown vegetation canopy: effect of crown shape and mutual shadowing," IEEE Trans. Geosci. Remote Sens. 30(2), 276-292 (1992).

20. J. L. Roujean, M. Leroy, and P.-Y. Deschamps, "A bidirectional reflectance model of the earth's surface for the correction of remote sensing data," J. Geophys. Res. Atmos. 97(D18), 20455-20468 (1992).

21. W. Wanner, X. Li, and A. Strahler, "On the derivation of kernels for kernel-driven models of bidirectional reflectance," J. Geophys. Res. Atmos. 100(D10), 21077-21089 (1995).

22. F. Maignan, F.-M. Bréon, and R. Lacaze, "Bidirectional reflectance of earth targets: evaluation of analytical models using a large set of spaceborne measurements with emphasis on the hot spot," Remote Sens. Environ. 90(2), 210-220 (2004).

23. A. Berk et al., Modtran4 User's Manual, Vol. 1731, p. 3010, Air Force Research Laboratory, Hanscom AFB, Massachusetts (1999).

24. B. Hapke et al., "The cause of the hot spot in vegetation canopies and soils: shadow-hiding versus coherent backscatter," Remote Sens. Environ. 58(1), 63-68 (1996).

25. X. Xiong et al., "MODIS on-orbit calibration and characterization," Metrologia 40(1), S89 (2003).

26. I. E. Vas, T. J. Kelly, and E. A. Scarl, "Space station reboost with electrodynamic tethers," J. Spacecr. Rockets 37(2), 154-164 (2000). 
27. L. Johnson and M. Herrmann, International Space Station Electrodynamic Tether Reboost Study, National Aeronautics and Space Administration, Marshall Space Flight Center, Springfield, Virginia (1998).

28. B. Hapke, Theory of Reflectance and Emittance Spectroscopy, Cambridge University Press, Cambridge (2012).

Rehman S. Eon received his BSc degree in mathematical physics and chemistry from Viterbo University in 2015. He is currently pursuing his $\mathrm{PhD}$ in imaging science at the Rochester Institute of Technology (RIT). His research interests include the use of optical remote sensing for the assessment of earth sediments and vegetation, radiative transfer modeling, and sensor calibration of Earth observing systems.

Biographies for the other authors are not available. 